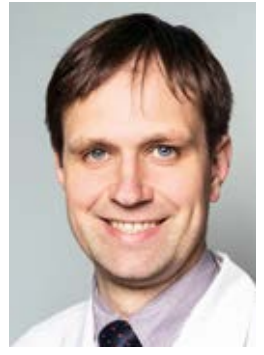

А. А. Аегтерев

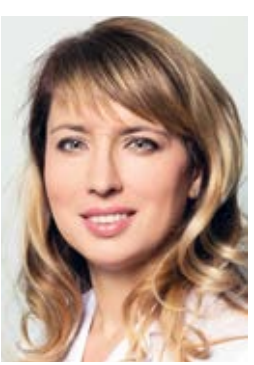

С.В. Быкова

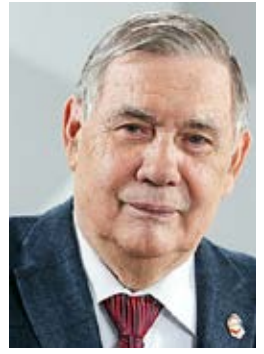

А.И. Пароренов

\section{Глютен-ассоциированная} головная боль

\author{
А. А. Аегтерев, К.М.Н., зав. неврологическим отАелением \\ С. В. Быкова, К.М.Н., зав. отАелением невоспалительной патологии кишечника \\ А. И. Парфенов, А.м.Н., профр., зав. отАелением патологии кишечника
}

ГБУЗ г. Москвы «Московский кАинический научно-практический центр имени
А.С. Аогинова» Аепартамента зАравоохранения г. Москвы

\title{
Gluten related headaches
}

D. A. Degterev, S. V. Bykova, A.I. Parfenov

A. S. Loginov Moscow Clinical Scientific Center, Moscow, Russia

Резюме

Целиакия и неассоциированная с целиакией чувствительность К гАютенУ явАяются расстройствами, Аиагностика которых бывает часто отложена на многие годы от первых кАинических проявлений заболевания. Характерным и более частым является Аебют целиакии с внекишечных проявлений или заболеваний, СвязаннЫХ С гАютенОМ. ОАним Из такиХ Состояний явАяетСя гоАовная боль. В статье преАставлены основные Аанные, известные к настоящему времени в отношении глютен-ассоциированной головной боли, вкАючая распространенность, КАинические проявления, особенности Аиагностики и результаты мечения.

КАючевые СлОва: целиакия; чувствительность к глютену; заболевания, связанные с гАютеном; головная бомь; мигрень.

\section{Summary}

Coeliac disease and non-celiac gluten sensitivity are disorders whose diagnosis can be delayed for many years from the first clinical manifestations. Typical debut of coeliac disease is an extraintestinal manifestation as gluten-related disorders. One of such impairments is headache. There are currently data about gluten-associated headache including prevalence, clinical manifestations, diagnostic features and treatment results in this paper. Key words: coeliac diseases, non-celiac gluten sensitivity, gluten-related disorders, headaches, migraine

\section{Глютен-ассоциированная головная боль}

Глютенчувствительная целиакия это генетически опосредованное заболевание, возникающее вследствие развития иммунной реакции в слизистой оболочке тонкого кишечника (СОТК) при ее контакте с белком глютеном (клейковиной) [1].

Диагностика целиакии основывается на клинической картине (диарея, вздутие живота, физическое недоразвитие, потеря массы тела, железодефицитная анемия и др.), серологических тестах (обнаружение в крови антител к глиадину, тканевой трансглутаминазе, эндомизию) и гистологических изменениях слизистой оболочки в биоптате, взятом из дистального отдела двенадцатиперстной кишки (атрофия ворсинок, гиперплазия крипт, лимфоцитарная инфильтрация эпителия). Лечение заключается в полном отказе от употребления глютенсодержащих продуктов - соблюдение аглютеновой диеты [2].
Распространенность целиакии в популяции колеблется в районе $1 \%$ $[3,4]$. При этом частота поражения нервной системы у пациентов с установленным диагнозом встречается в $10,0-22,5 \%$ случаев $[5,6]$ и даже достигает $42,0 \%$ у нелеченых пациентов [7].

Важным, с точки зрения диагностики и выбора тактики лечения неврологических осложнений целиакии, является определение ее клинических форм или вариантов. Классификация основывается на клинических проявлениях заболевания и данных лабораторно-инструментальной диагностики.

Согласно принятому в 2012 году в г. Осло (Норвегия) консенсусу по определению диагноза целиакии и связанным с ним терминами [8] в модификации B. Lebwohl и соавт. [3], целиакию можно классифицировать на следующие клинические формы:
- потенциальная - клинических проявлений не определяется при наличии положительных серологических тестов и неизменной СОТК при биопсии;

- бессимптомная - отсутствие каких-либо симптомов при наличии положительных серологических тестов и изменений СОТК при биопсии;

- классическая - основным клиническим проявлением является диарея с признаками мальабсорбции при наличии положительных серологических тестов и изменений СОТК при биопсии;

- неклассическая - присутствие внекишечных проявлений (неврологических расстройств, анемии, остеопороза и т.п.) при наличии положительных серологических тестов и изменений СОТК при биопсии;

- рефрактерная-наличие стойких клинических симптомов и атро- 
фия ворсинок тонкой кишки при биопсии, несмотря на соблюдение аглютеновой диеты;

- симптоматическая - собирательное определение, объединяющее любые клинические формы (бессимптомная, классическая, неклассическая, рефрактерная).

Утвержденный в 2016 году Всероссийский консенсус по диагностике и лечению целиакии У детей и взрослых берет за основу классификацию, разработанную в 2005 году в Центральном научно-исследовательском институте гастроэнтерологии (ЦНИИГ) [9]. В ней используются схожие с предыдущей классификацией определения клинических форм целиакии, однако вместо терминов «классическая» и «неклассическая» используются «типичная» и «атипичная» соответственно.

Кроме «целиакии», в научной и популярной литературе используются такие термины, как «непереносимость глютена» и «чувствительность к глютену». Появление данных нозологических форм было обусловлено растущим количеством пациентов, у которых присутствуют разнообразные клинические симптомы, исчезающие на фоне исключения глютена из рациона [10].

Под непереносимостью глютена обычно подразумевается такое состояние, когда у пациента с желудочно-кишечными расстройствами в отсутствие лабораторных и гистологических признаков целиакии отмечается клиническое улучшение после начала аглютеновой диеты $[11,12,13]$. Однако в настоящее время считается, что термин «непереносимость глютена» не является конкретным и несет в себе терминологическое противоречие. Глютен может стать причиной диспепсических нарушений не только за счет запуска дизимунной реакции в стенке тонкой кишки, что происходит при целиакии, но и действуя напрямую, обладая рядом свойств, характерных для группы белков лектинов (увеличивая проницаемость кишечной стенки, вызывая агглю- тинацию эритроцитов). Учитывая это, рекомендовано вместо термина «непереносимость глютена» использовать другой - «заболевания, связанные с глютеном» (gluten-related disorders) $[8,14]$.

Другой термин «чувствительность к глютену» используется авторами по-разному. Или как клинические проявления целиакии [15], или как любое заболевание, ассоциированное с целиакией (глютеновая атаксия, герпетиформный дерматит) [16, 17], или как состояние, при котором симптомы энтеропатии возникают при приеме глютена в отсутствие антител к тканевой трансглутаминазе и эндомизию и возможном присутствии антител к глиадину и генетической предрасположенности к целиакии [18]. Ввиду широкой трактовки термина «чувствительность к глютену» рекомендовано к использованию другое определение - «неассоциированная с целиакией чувствительность к глютену» (non-celiac gluten sensitivity, NCGS), что подразумевает под coбой появление одного или нескольких изменений на клиническом, иммунологическом или морфологическом уровнях при употреблении в пищу глютенсодержащих продуктов людьми, у которых ранее была исключена целиакия $[8,18]$.

Неврологические нарушения могут выявляться как у пациентов с целиакией, так и при наличии неассоциированной с ней чувствительности к глютену (НЦЧГ). В свою очередь, целиакия с неврологическими расстройствами может протекать как классическая (типичная), осложнившаяся внекишечными симптомами, или как неклассическая (атипичная). У пациентов НЦЧГ при неврологической манифестации расстройства обычно выявляются антитела к глиадину класса IgG и (или) IgA+/- антитела к тканевой трансглутаминазе или эндомизию, но в отсутствии гистологических изменений в СОТК [19].

Механизмы повреждения нервной системы при глютеновой энтеропатии включают перекрестную иммунную реакцию, отложение иммунных комплексов, прямую нейротоксичность и дефицит витаминов или питательных веществ [7, 20].
Для целиакии и НЦЧГ характерна ассоциация с разнообразными неврологическими и психоэмоциональными расстройствами [21]. К основным синдромам поражения нервной системы при целиакии относятся глютеновая атаксия и полиневропатия. Однако спектр неврологических проявлений целиакии гораздо шире и включает в себя такие состояния и симптомы, как эпилептические приступы, повышенную утомляемость, головокружение, головную боль, повышенный уровень тревожности, депрессию, синдром раздраженного кишечника, сонливостью, мышечные боли, напоминающие фибромиалгию, шизофрению [10, 22].

Общая оценочная распространенность головной боли у взрослых с целиакией составляет 26\% [23], что немного выше, чем распространенность головной боли среди детей с целиакией, которая равна 18,3\% [24]. По данным мета-анализа четырех контролируемых исследований, головная боль выявлялась в 7,3 раза чаще среди пациентов с целиакией, чем в общей популяции [23].

Головная боль, особенно головная боль мигренозного типа, часто является манифестным проявлением неклассической (атипичной) целиакии. Мигренозная головная боль при целиакии имеет высокую коморбидность с другими неврологическими нарушениями. Среди 40 взрослых пациентов с целиакией с симптомами и признаками дисфункции центральной нервной системы (преимущественно глютеновая атаксия) у всех пациентов была диагностирована эпизодическая мигренеподобная головная боль [25].

Интенсивность головной боли, ассоциированной с целиакией, обычно бывает выше, чем в общей популяции, что заставляет пациентов активно искать медицинской помощи и может стать причиной подозрения о наличии целиакии у такого пациента [26]. Основными клиническими формами головной боли, ассоциированной с целиакией, являются мигрень и головная боль напряжения. Напротив, такие формы головной боли, как кластерная головная боль, гемикрания конти- 
нуа и тригеминальная невралгия, не характерны при целиакии [27].

Gabrielli и соавт. с целью оценки распространенности идиопатической мигрени сравнили 90 пациентов с целиакией с донорами крови. Было выявлено 10-кратное превосходство встречаемости головной боли при целиакии по сравнению с практически здоровыми людьми $(4,4$ и $0,4 \%$ соответственно) [28].

Исследований, изучающих распространенность головной боли у пациентов с неассоциированной с целиакией чувствительностью к глютену (НЦЧГ), существенно меньше, чем при целиакии. Головная боль при НЦЧГ определяется при употреблении в пищу глютена. При исследовании 486 пациентов с НЦЧГ (взрослых и детей) головная боль определялась в $54 \%$ случаев [10]. В другой работе оценивали когорту из 78 детей с НЦЧГ, среди которых головная боль наблюдалась в $32 \%$ случаев [29].

Рутинные диагностические исследования, такие как электроэнцефалограмма (ЭЭГ), при головной боли, ассоциированной с целиакией, обычно ничего не выявляют. Однако описаны находки при специальных методах исследования.

Редкой, но характерной находкой при компьютерной томографии (КТ) головы можно считать наличие кальцинатов в головном мозге. Отложения кальция обычно локализуются в затылочных отделах. Характерным для целиакии является сочетание кальцинатов головного мозга с эпилептическим синдромом, что носит название синдрома эпилепсии и церебральной кальцификации (epilepsy and cerebral calcification syndrome, CEC) [19]. В литературе описаны случае наличия кальцинатов головного мозга с мигренеподобной головной болью как в комбинации с признаками эпилепсии или эпилептиформной активности на ЭЭГ, так и без нее [23].

При магнитно-резонасной томографии (МРТ) головного мозга взрослого пациента с целиакией выявляется повреждение белого вещества в $36 \%$ случаев [30]. Среди детей каждый шестой пациент с целиакией и головной болью имел изменения в белом веще- стве головного мозга при МРТ [23]. Alehan et al. сообщили, что изменения в белом веществе головного мозга присутствовали у каждого четвертого ребенка с мигренозной головной болью и антителами к тканевой трансглутаминазе в крови [31].

Методом однофотонной эмиссионной компьютерной томографии (ОФЭКТ, SPECT) у пациентов с целиакией и мигренозной головной болью выявлено нарушение регионального кровотока (гипоперфузия) в коре головного мозга. Характерно, что таких изменений не регистрируется у пациентов с мигренью без целиакии [28], в то время как методом позитронно-эмиссионной компьютерной томографии (ПЭТ КТ) на четырех детях с целиакией не было выявлено изменений церебрального кровотока [32].

В многочисленных исследованиях показано, что применение аглютеновой диеты снижало интенсивность и частоту приступов головной боли при целиакии [7, 27, 33]. Показано снижение частоты головной боли на фоне аглютеновой диеты у взрослых в 51,6-100,0\%, у детей - в 69,2100,0 случаев. Полное исчезновение головной боли в случае приверженности к аглютеновой диете наблюдалось у 75,0\% взрослых и 71,3\% детей [23].

Соблюдение строгой аглютеновой диеты сопровождалось нормализацией церебрального кровотока, по данным ОФЭКТ [28], снижением интенсивности образования очагов в белом веществе головного мозга, по данным МРТ, при сохранности старых очагов и кальцинатов в головном мозге [30].

\section{Заключение}

Головная боль, ассоциированная с целиакией, является одним из основных внекишечных проявлений этого заболевания. Выраженность симптомов, резистентность к традиционным методам лечения, наличие изменений, по данным нейровизуализации, и хороший ответ на применение аглютеновой диеты, подчеркивают важность как можно более ранней диагностики и начала лечения данного типа головной боли.

\section{Выводы}

1. Пациенты с головной болью при наличии кальцификации в затылочной или теменно-затылочной областях головного мозга должны быть обследованы для исключения целиакии;

2. Пациенты с головной болью, у которых, по данным МРТ, определяются неспецифические изменения в белом веществе головного мозга, должны быть обследованы для исключения целиакии;

3. Требуется проведение дальнейших исследований для определения диагностической ценности измерения церебрального кровотока при головной боли, ассоциированной с целиакией;

4. У пациентов с целиакией и НЦЧГ, придерживающихся аглютеновой диеты, присутствует риск случайного попадания следов глютена в пищу, что может привести к появлению головной боли, поэтому рекомендуется проводить регулярные исследования уровня антител к тканевой трансглутаминазе и глиадину в крови;

5. Определение антител к глиадину является единственным скриннинговым методом оценки активности НЦЧГ.

\section{Список митературы}

1. Парфренов А.И. Энтерология: Руководство А^я врачей. 2-е изА. М.: МИА, 2009.

2. Парфенов А.И. Целиакия. Эволюция преАставлений о распространенности, кАинических проявАениях и значимости этиотропной терапии: руководство / А.И. Парфренов.-М.: Aнахарсис, 2007.- 376 с.

3. Benjamin Lebwohl, David S Sanders, Peter HR Green. Lancet 2018; 391: 70-81.

4. West J, Logan RFA, Hill PG, et al. Seroprevalence, correlates, and characteristics of undetected celiac disease in England. Gut 2003; 52: 960-65.

5. Holmes GKT. Neurological and psychiatric complications in coeliac disease. In: Gobbi G, Anderman F, Naccarato S, Banchini G, eds. Epilepsy and other neurological disorders in coeliac disease. London: John Libbey, 1997: 251-64.

6. Briani C, Zara G, Alaedini A, et al. Neurological complications of coeliac disease and autoimmune mechanisms: a prospective study. J Neuroimmunol. 2008; 195: 171-75.

7. Hadjivassiliou M, Croall ID, Zis $P$, et al. Neurologic deficits in patients with newly diagnosed celiac disease are frequent and linked with autobodies to transglutaminase 6. Clin Gastroenterol Hepatol. 2019.

8. Ludvigsson JF, Leffler DA, Bai JC et al. The Oslo definitions for coeliac disease and related terms. Gut. 2013; 62 (1):43-52.

9. Всероссийский консенсус по Аиагностике илечению целиакии у детей и взрослых. При- 
нят на 42-й научной сессии ЦНИИГ (2-3 марта 2016 г.) Consilium Medicum. Педиатрия 2016, № 1, стр. 6-19.

10. Volta U, Bardella MT, Calabr A et al. Study Group for Non-Celiac Gluten Sensitivity. An Italian prospective multicenter survey on patients suspected of having non-celiac gluten sensitivity. BMC Med, 2014, 12: 85

11. Bardella MT, Fredella C, Saladino V, et al. Gluten intolerance: gender- and age-related differences in symptoms. Scand I Gastroenterol 2005; 40: $15 \mathrm{e} 19$.

12. Poloni N, Vender S, Bolla E, et al. Gluten encephalopathy with psychiatric onset: case report. Clin Pract Epidemiol Ment Health 2009; 5: 16.

13. Cascella NG, Kryszak D, Bhatti B, et al. Prevalence of celiac disease and gluten sensitivity in the United States clinical antipsychotic trials of intervention effectiveness study population. Schizophr Bull 2011; 37: 94e100.

14. Helms S. Celiac disease and gluten-associated diseases. Altern Med Rev 2005; 10: 172e92.

15. Marsh MN. Gluten, major histocompatibility complex, and the small intestine. A molecular and immunobiologic approach to the spectrum of gluten sensitivity ('celiac sprue'). Gastroenterology 1992; 102: 330e54.

16. Sardy M, Karpati S, Merkl B, et al. Epiderma transglutaminase (TGase 3) is the autoantigen of dermatitis herpetiformis. J Exp Med 2002; 195: 747e57.

17. Hadjivassiliou M, Grunewald RA, Chattopadhyay AK, et al. Clinical, radiological, neurophysiological, and neuropathological characteristics of gluten ataxia. Lancet 1998; 352: 1582e5.
18. Massari S, Liso M, De Santis L, et al. Occurrence of nonceliac gluten sensitivity in patients with allergic disease. Int Arch Allergy Immunol 2011; 155: 389e94.

19. Julian T., Hadjivassiliou M., Zis P. Gluten sensitivity and epilepsy: A systematic review. J. Neurol. 2018.

20. Pennisi M, Bramanti A, CantoneM, et al. Neurophysiology of the "Celiac Brain": disentangling gut-brain connections. Front Neurosci. 2017; 11: 498.

21. Пароренов А.И. Неврологические и психи ческие нарушения у больных гАютенчувствительной целиакией. Consilium Medicum. При ^ожение Гастроэнтерология. 2009; 1: 46-50.

22. Nijeboer P., Bontkes H. J., Mulder C. J.J., Bouma $G$. Non-celiac gluten sensitivity. Is it in the gluten or the grain? J. Gastrointestin. Liver Dis. 2013; 22 (4): 435-40. PMID: 24369326.

23. Zis $P$, Julian T, Hadjivassiliou M. Headache associated with coeliac disease: a systematic review and meta-analysis. Nutrients. 2018; 10: 1445.

24. Sansotta N, Amirikian K, Guandalini S, et al. Celiac disease symptom resolution: effectiveness of the gluten-free diet. J Pediatr Gastroenterol Nutr. 2018; 66: 48-52.

25. Hadjivassiliou M., Grünewald R.A., Lawden M. Davies-Jones G.A., Powell T., Smith C.M. Headache and CNS white matter abnormalities associated with gluten sensitivity. Neurology 2001, 56, 385-388.

26. Therrien A., Kelly C.P., Silvester J. A., Celiac disease extraintestinal manifestation and associated conditions. J Clin Gastroenterol Volume 54, Number 1, January 2020
27. Dimitrova AK, Ungaro RC, Lebwohl B, et al. Prevalence of migraine in patients with celiac disease and inflammatory bowel disease. Headache. 2013; 53: 344-355.

28. Gabrielli M. Cremonini F. Fiore G. et. al. Association between migraine and Celiac disease: Results from a preliminary case-control and therapeutic study. Am. J. Gastroenterol. 2003, $98,625-629$.

29. Volta U., Tovoli F., Cicola R., Parisi C., Fabbri A Piscaglia M., Fiorini E., Caio G. Serological tests in gluten sensitivity (nonceliac gluten intolerance) J. Clin. Gastroenterol. 2012

30. Currie S., Hadjivassiliou M., Clark M. J.R., Sanders D.S., Wilkinson I.D., Griffiths P.D., Hoggard N. Should we be "nervous" about coeliac disease? Brain abnormalities in patients with coeliac disease referred for neurological opinion. J. Neurol. Neurosurg. Psychiatry 2012, 83, 1216-1221.

31. Alehan F., Ozçay F., Erol I., Canan O., Cemil T. Increased risk for coeliac disease in paediatric patients with migraine. Cephalalgia 2008 28, 945-949.

32. Lionetti E., Francavilla R., Maiuri L., Ruggieri M. Spina M., Pavone P., Francavilla T., Magistà A. M. Pavone L. Headache in Pediatric Patients With Celiac Disease and Its Prevalence as a Diagnostic Clue. J. Pediatr. Gastroenterol. Nutr. 2009 49, 202-207.

33. Амитриева Ю. А., Захарова И.Н. Эоффектив ность безглютеновой Аиеты при невроло гических заболеваниях и психиатрических расстройствах: мифо или реальность? // МеАицинский совет.- 2018, № 2, стр. 156-161.
Аия цитирования: Аегтерев А. А. Быкова С. В. Парсенов А. И. Глютен-ассоциированная головная боль. МеАицинский алфравит. 2020; (1):36-39. https://doi. org/10.33667/2078-5631-2020-1-36-39
For citation: Degterev D. A., Bykova S. V., Parfenov A.I. Gluten related headaches. Medical alphabet.2020; (1):36-39. https://doi.org/10.33667/2078-5631-2020-1-36-39

\section{$\mathbf{Y}$ \\ ФГАОУ ВО РНИМУ \\ им. Н.И. Пирогова Минздрава России \\ Кафедра факультетской терапии \\ им. акад. А.И. Нестерова}

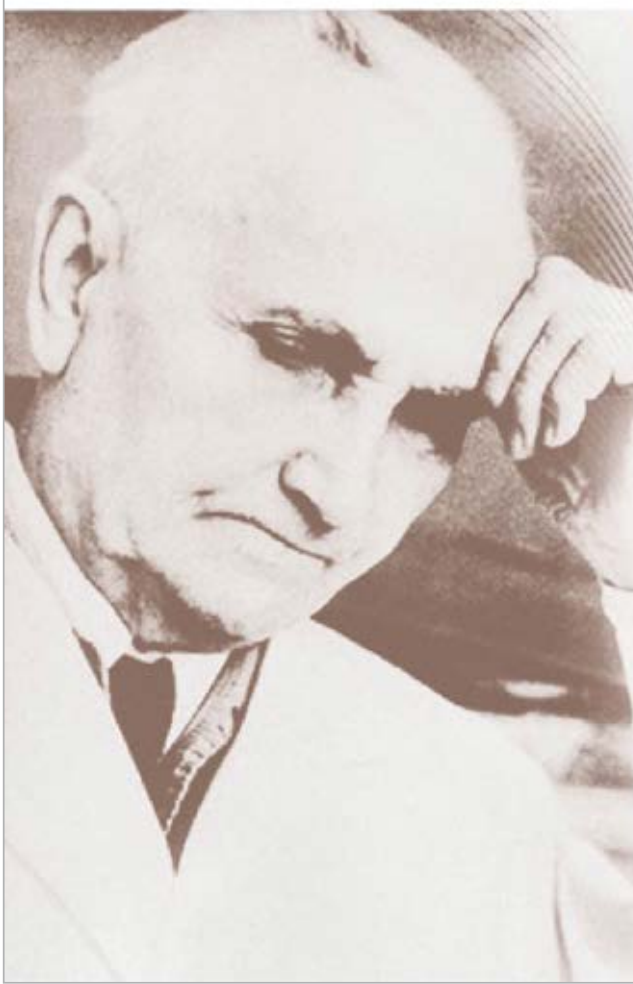

VIII ЮБИЛЕЙНАЯ

НАУЧНО-ПРАКТИЧЕСКАЯ КОНФЕРЕНЦИЯ С МЕЖДУНАРОДНЫМ УЧАСТИЕМ

Посвященная 110-летию кафедры

факультетской терапии

им. академика А.И. Нестерова

\section{«НЕСТЕРОВСКИЕ ЧТЕНИЯ»}

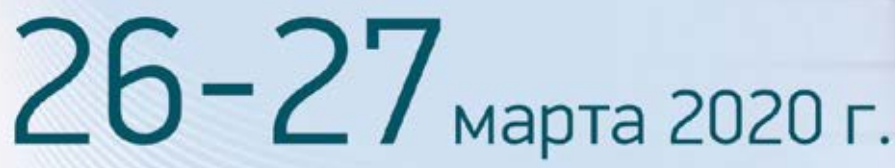

Москва, Президент-Отель, ул. Б. Якиманка, д. 24

www.medkongress.ru 